\title{
May Nesfatin-1 be a Biomarker in Acute Mesenteric Ischemia?
}

\author{
Cihad Tatar¹, Fatih Alper Ahlatci², Ufuk Oguz Idiz¹, Ali Emre Nayci, Said Incir33, Orhan Agcaoglu4, Cemile Idiz ${ }^{5}$ \\ and Emre Balik4 \\ IDepartmet of General Surgery, Istanbul Training and Research Hospital, Istanbul, Turkey \\ 2Department of Pathology, Corlu State Hospital, Tekirdag, Turkey \\ 3 Department of Biochemistry, Koc University, Medical Faculty, Istanbul, Turkey \\ ${ }^{4}$ Department of General Surgery, Koc University, Medical Faculty, Istanbul, Turkey \\ 5 Department of Internal Medicine, Istanbul University, Istanbul Medical Faculty, Istanbul, Turkey
}

\begin{abstract}
Objective: To investigate the diagnostic value of nesfatin-1 in cases of intestinal ischemia and ischemia/reperfusion.

Study Design: An experimental study.

Place and Duration of Study: The Experimental Animals Laboratory of Bezmialem University, in June 2018.

Methodology: Twenty-one healthy male Sprague Dawley rats were randomly divided into three groups of 7 rats each. In group 1: 1-hour intestinal ischemia followed by 5-hour reperfusion was performed. In group 2: rats were subjected to 6-hour intestinal ischemia. In group 3: rats underwent laparotomy and closure without performing any further procedure. Changes in leukocyte count, amylase, blood sugar, LDH, SGOT, CRP, and nesfatin-1 levels were determined. For histopathological examination, a small intestinal sample was taken and preserved in $10 \%$ formaldehyde.

Results: Nesfatin-1 value in group 2 was significantly higher than that in group 1 and group 3 ( $p=0.005$, and $p<0.001$ respectively). Nesfatin-1 value in group 1 was significantly higher than that in group 3 . A significant $(r=0.864 / p<0.001)$ positive correlation was observed between nesfatin-1 value and pathology score. The pathology score of group 2 was significantly higher than that of group 1 and group $3(p<0.001)$.

Conclusion: Serum nesfatin-1 can be a biomarker in acute mesenteric ischemia.
\end{abstract}

Key Words: Acute, Biomarker, Intestinal, Mesenteric Ischemia, Nesfatin-1, Reperfusion.

How to cite this article: Tatar C, Ahlatci FA, Idiz UO, Nayci AE, Incir S, Agcaoglu O, Idiz C, Balik E. May Nesfatin-1 be a biomarker in acute mesenteric ischemia? J Coll Physicians Surg Pak 2019; 29(10):928-31.

\section{INTRODUCTION}

Acute mesenteric ischemia (AMI) is a clinical condition caused by sudden inadequate blood flow through any small intestinal segment accompanied by ischemia, cellular damage, and intestinal necrosis and can be lifethreatening, if not treated. AMI is a rare cause of abdominal pain with a low overall incidence of approximately $0.09 \%-0.2 \%$ of patients admitted to the emergency department. ${ }^{1}$

In AMI, there is severe abdominal pain that is nonproportional to the physical examination findings. In most cases, intestinal ischemia progresses transmurally and is diagnosed after peritonitis and sepsis develop. Certain imaging methods are also performed in case of clinical suspicion. X-ray imaging and abdominal ultrasonography have very limited diagnostic value, and abdominal computed tomography has low sensitivity and specificity. Contrast-enhanced CT may be used as the first-line imaging technique because of its excellent

Correspondence to: Cihad Tatar, Department of General Surgery, Istanbul Training and Research Hospital, Istanbul, Turkey

E-mail: tatarcihad@gmail.com

Received: February 08, 2019; Revised: May 20, 2019;

Accepted: July 09, 2019 reported sensitivity and specificity. ${ }^{2}$ The American College of Gastroenterology guidelines define angiography as the gold standard in the diagnosis of mesenteric ischemia. ${ }^{3}$ However, the disadvantages of catheter angiography are: it is an invasive and timeconsuming technique, and many hospitals are unable to perform it. Recent studies have reported that computed tomography angiography is a less invasive and less time-consuming method and suggested that it can be used as the gold standard for mesenteric ischemia diagnosis with its $96 \%$ sensitivity and $94 \%$ specificity. 4,5 According to the guidelines of the World Society of Emergency Surgery, there are no laboratory studies that are sufficiently accurate to identify the presence or absence of ischemic or necrotic bowel, although elevated L-lactate and D-dimer levels may be indications (Recommendation 1B). Although biomarkers such as intestinal fatty acid-binding protein, serum alphaglutathione S-transferase, and cobalt-albumin binding assay are reported as auxiliary tools for diagnosis, there are still no definite biomarkers. ${ }^{1}$

Nesfatin-1, first discovered by Oh-l et al. in 2006, is an appetite-controlling peptide consisting of 82 amino acids and present in many nuclei of the hypothalamus, including the paraventricular nucleus. ${ }^{6}$ Metabolism that produces cytokines, such as IL-1 and tumor necrosis 
alpha, and adipose tissue, which is an important mediator of inflammation, also produce adipokines such as leptin, adiponectin, resistin, and visfatin. ${ }^{7}$ Nesfatin-1 is a recently discovered adipokine and is associated with obesity and metabolic syndrome. Studies have demonstrated the effects of nesfatin-1 on feeding behaviour, neuroendocrine regulation, autonomic control of visceral functions, development and differentiation of adipose tissue, inflammation, thermoregulation, pancreatic insulin secretion, glucose homeostasis in KC, sleep, attention, anxiety, and stress. In addition, it has also been reported to regulate gastric emptying, gastric acid secretion, gastric motility, and reproductive functions. ${ }^{8-11}$

The objective of this study was to investigate the diagnostic value of nesfatin-1 in cases of intestinal ischemia and ischemia/reperfusion ( $(/ R)$, which have not been previously studied in the literature.

\section{METHODOLOGY}

This experimental study was carried out at the Experimental Animals Laboratory of Bezmialem University, in June 2018 and approved by the Ethics Committee, Faculty of Medicine, Bezmialem University. In the study, 21 male Sprague Dawley rats weighing 370-480 g (standard pelleted diet) were randomly divided into three groups of 7 rats each.

In group 1, superior mesenteric artery (SMA) and superior mesenteric vein (SMV) were isolated by laparotomy, silk sutures were used for knotting, and the abdomen was closed. After 1 hour of ischemia, silk sutures on SMA and SMV were removed through relaparatomy under anesthesia, and 5 hours of reperfusion was allowed (Figure 1). In group 2, laparatomy was performed on the rats. Silk sutures were used for the knotting of SMA and SMV (exposed to 6-hour ischemia), and the laparotomy opening was closed (Figure 2). In group 3, laparatomy was performed, SMA and SMV were isolated, and the abdomen was closed without performing any further procedure (Figure 3 ).

After 6 hours, relaparatomy was performed under anesthesia in all animals, and pulsatility of SMA was assessed in the $\mathrm{I} / \mathrm{R}$ group. Absence of pulsation was considered as an exclusion criterion. Then, intracardiac blood samples were collected from all rats for biochemical examination; leukocyte count, amylase, blood sugar, LDH, SGOT, CRP, and nesfatin-1 levels were measured; then rats were killed. For histopathological examination, a small intestinal sample was taken and preserved in $10 \%$ formaldehyde.

Histopathological examination was performed by a pathologist who was blinded to the group assignments of the numbered samples. Microscopic examination was performed based on the scale developed by Chiu et al. using hematoxylin-eosin staining method (Table I). ${ }^{12}$

\begin{tabular}{ll} 
Table I: $H$ Histopathological examination. \\
\hline Grade & Description \\
\hline 0 & Normal mucosal villi. \\
1 & Slight elevation of epithelium from lamina propria at the apex of villi. \\
2 & Moderate elevation of epithelial layer from lamina propria. \\
3 & Massive epithelial lifting down the sides of villi. \\
4 & Denuded villi with lamina propria exposed and dilated capillaries. \\
5 & Disintegration.
\end{tabular}

The SPSS 22.0 software was used for statistical analyses, and mean, standard deviation, median, minimum-maximum, frequency, and ratios were used in descriptive statistics. Distribution of the variables was tested using the Kolmogorov-Smirnov test. The One-Way ANOVA was used for the analysis of continuous independent variables and; if there was a statistical difference, post-hoc test was performed to find which group caused the difference. Also Kruskal-Wallis test used for ordinal independent variables. Spearman correlation test was used for the pathological and nesfatin value correlation and the statistical significance level was accepted as $p<0.05$.

\section{RESULTS}

Mean pathology scores were $1.3 \pm 1.3$ in group $1,4.4$ \pm 0.5 in group 2 , and $0 \pm 0$ in group 3 . Images of grades 0,1 , and 3 under $100 \times$ magnification with hematoxylineosin staining are shown in Figure 4. Mean nesfatin-1 values were $74.9 \pm 30.3$ in group $1,137.1 \pm 42$.3 in group 2, and $35.7 \pm 18.9$ in group 3 .

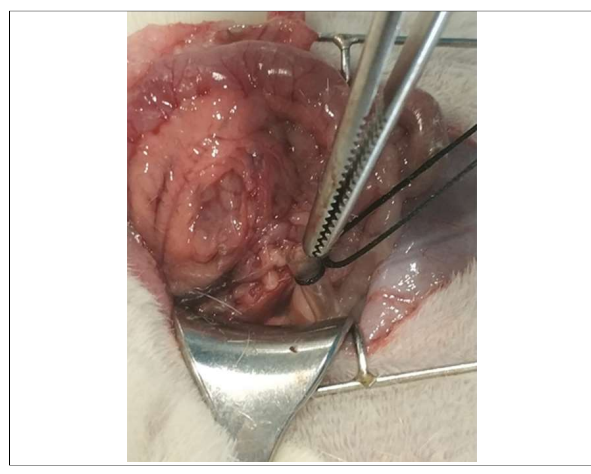

Figure 1: SMA and superior mesenteric vein (SMV) were isolated by laparotomy, silk sutures were used for knotting.

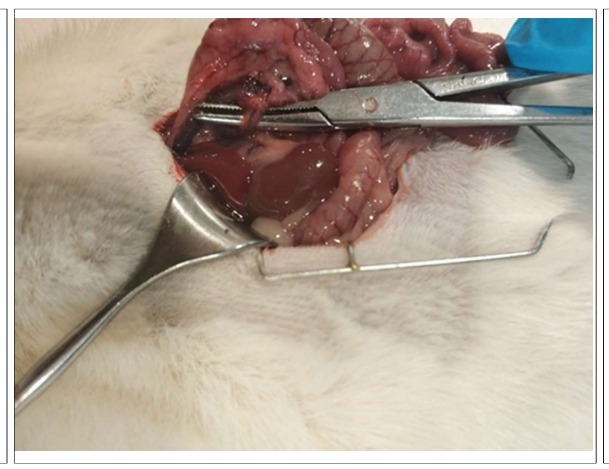

Figure 2: Isolation of SMA and superior mesenteric vein (SMV).

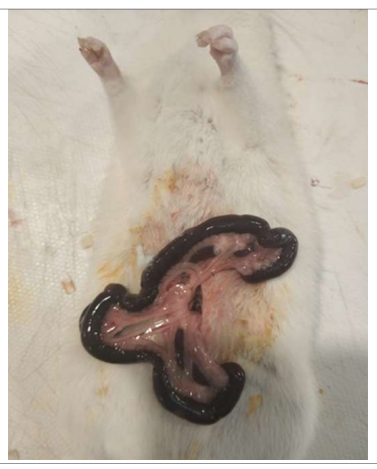

Figure 3: Ischemic intestines. 


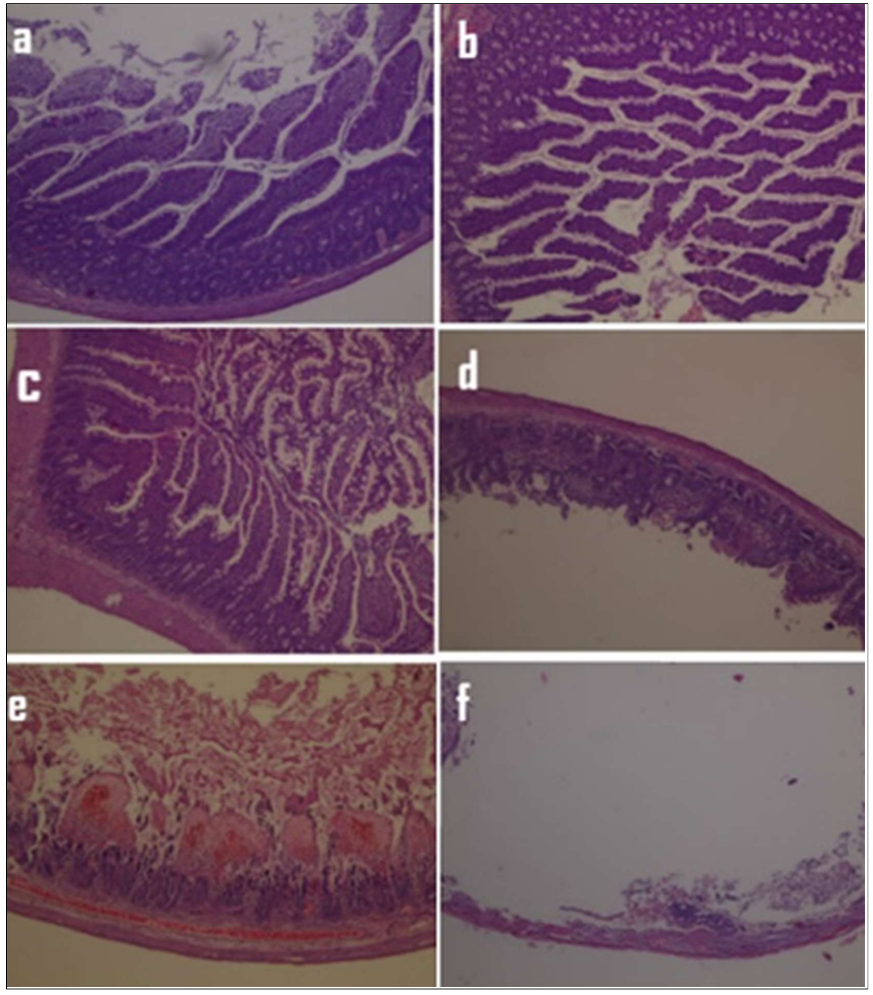

Figure 4: (a) Grade 0 (Hematoxylin-eosin, $100 \mathrm{x}$ )

(b) Grade 1 Hematoxylin-eosin, $100 \mathrm{x}$ )

(c) Grade 2 Hematoxylin-eosin, $100 x$ )

(d) Grade 3 Hematoxylin-eosin, $100 x$ )

(e) Grade 4 Hematoxylin-eosin, $100 x$ )

(f) Grade 5 Hematoxylin-eosin, $100 x$ )

The pathology score of group 2 was significantly higher than that of group 1 and group $3(p<0.001)$.

Nesfatin-1 value in group 2 was significantly higher than that in group 1 and group 3 at the post-hoc test (respectively $p=0.005, p<0.001$ ). Nesfatin- 1 value in group 1 was significantly higher than that in group 3 $(p=0.026)$.

A significant $(r=0.864 / p<0.001)$ positive correlation was observed between nesfatin-1 value and pathology score.

\section{DISCUSSION}

The mortality rates of AMI varying between $30 \%$ and $100 \%$ due to the absence of a specific diagnostic method for AMI.13-17 However, the absence of a biomarker for early diagnosis prevents the reduction of mortality rate.

Reperfusion is defined as the re-establishment of blood flow after ischemia, and this is necessary to prevent irreversible cell damage. In recent years, studies reporting nesfatin- 1 as a protective peptide in cases of cardiac, renal, and intestinal ischemia have been published. ${ }^{18-20}$ Dai et al. compared patients with acute myocardial infarct and patients with angina pectoris with a control group and found significantly lower nesfatin-1 plasma levels in the acute myocardial infarct group. ${ }^{21}$ The statistical analysis of our study revealed significantly higher nesfatin-1 values in the ischemia group than in the other groups. In addition, the I/R group had significantly higher nesfatin-1 values than did the control group.

Intraperitoneal administration of nesfatin-1 was shown to have anti-inflammatory effect in acedic acid-induced gastritis model. ${ }^{22}$ Researchers have stated that nesfatin-1 had this effect through the inhibition of pro-inflammatory mediators as well as the establishment of a balance between oxidant and antioxidant systems. Ozturk et al. suggested in their ischemic colitis model that nesfatin-1 can exhibit an anti-inflammatory effect by preventing neutrophil infiltration into the tissue and by inhibiting free radical formation, and they added that it can have an antioxidant effect on oxytocin and ghrelin receptors and colitis. ${ }^{23}$ Ayada et al. investigated the effects of chronic systemic nesfatin-1 administration on microcirculation effectors and oxidant-antioxidant states in an intestinal $\mathrm{I} / \mathrm{R}$ model designed in rats. ${ }^{20}$

In this study, nesfatin-1 balanced the oxidative state by decreasing the eNOS level and inhibiting the NO production.

Although this study revealed a positive correlation between nesfatin-1 values and pathology score, the causative mechanism could not be clearly understood. No definite data was obtained as to whether nesfatin-1 is produced as a result of an inflammation process or increased as a result of an anti-inflammatory response. In line with these findings, it can be interpreted that nesfatin-1 leads to an anti-inflammatory effect by inhibiting pro-inflammatory cytokines and establishes a balance in the oxidant-antioxidant system and can be a biomarker in acute mesenteric ischemia. Further studies are required on this aspect. Studies have suggested an association between nesfatin-1 and many diseases, and different mechanisms were addressed. The limitations of this study include its experimental design and limited number of rats.

\section{CONCLUSION}

In this study, nesfatin-1 levels were significantly higher in the $\mathrm{AMI}$ and $\mathrm{I} / \mathrm{R}$ groups.

\section{ETHICAL APPROVAL:}

Ethical pproval from the Ethics Committee of Bezmialem University was obtained prior to initiation of the study.

\section{CONFLICT OF INTEREST:}

Authors declared no conflict of interest.

\section{AUTHORS' CONTRIBUTION:}

\section{CT: Consept, design, resource.}

FAA, UOI, AEN, SI, OA, CI, EB: Materials, data collection and/or processing, analysis and Interpretation, literature search, critical reviews. 


\section{REFERENCES}

1. Bala M, Kashuk J, Moore EE, Kluger Y, Biffl W, Gomes CA, et al. Acute mesenteric ischemia: Guidelines of the World Society of Emergency Surgery. World J Emerg Surg 2017; 12:38-49.

2. Copin P, Zins M, Nuzzo A, Purcell $Y$, Beranger-Gibert $S$, Maggiori $L$, et al. Acute mesenteric ischemia: A critical role for the radiologist. Diagn Interv Imaging 2018; 99:123-34.

3. Brandt LJ, Boley SJ. AGA technical review on intestinal ischemia. American Gastrointestinal Association. Gastroenterol 2000; 118:954-68.

4. Kirkpatrick ID, Kroeker MA, Greenberg HM. Biphasic CT with mesenteric CT angiography in the evaluation of acute mesenteric ischemia: Initial experience. Radiology 2003; 229:91-8.

5. Wyers MC. Acute mesenteric ischemia: Diagnostic approach and surgical treatment. Semin Vasc Surg 2010; 23:9-20.

6. Oh-I S, Shimizu H, Satoh T, Okada S, Adachi S, Inoue K, et al. Identification of nesfatin-1 as a satiety molecule in the hypothalamus. Nature 2006; 443:709-12.

7. Kershaw EE, Flier JS. Adipose tissue as an endocrine organ. J Clin Endocrinol Metab 2004; 89:2548-56.

8. Stengel A, Tache Y. Nesfatin-1 role as possible new potent regülatör of food intake. Regul Pept 2010; 163:18-23.

9. Stengel A, Goebel M, Yakubov I, Wang L, Witcher D, Coskun T, et al. Identification and characterization of nesfatin-1 immunoreactivity in endocrine cell types of the rat gastric oxyntic mucosa. Endocrinol 2009; 150:232-8.

10. Ramanjaneya M, Chen J, Brown JE, Tripathi G, Hallschmid M, Patel $\mathrm{S}$, et al. Identification of nesfatin-1 in human and murine adipose tissue: A novel depot-specific adipokine with increased levels in obesity. Endocrinol 2010; 151:3169-80.

11. Aydin S. Multi-functional peptide hormone NUCB2/nesfatin-1. Endocrine 2013; 44:312-25.

12. Chiu CJ, Mcardle AH, Brown R, Scott HJ, Gurd FN. Intestinal mucosal lesion in low-flow states. Arch Surg 1970; 101:478-83.
13. Kassahun WT, Schulz T, Richter $\mathrm{O}$, Hauss J. Unchanged high mortality rates from acute occlusive intestinal ischemia: Six year review. Langenbecks Arch Surg 2008; 393:163-71.

14. Tsai CJ, Kuo YC, Chen PC, Wu CS. The spectrum of acute intestinal vascular failure: A collective review of 43 cases in Taiwan. Br J Clin Pract 1990; 44: 603-5.

15. Clark ET, Gerwitz BL. Mesenteric ischemia. In: Hall JB, Schmidt GA, Wood LD, Eds. Principles of Critical Care. New York, NY: McGraw-Hill; 1998: p.1279-86.

16. Aliosmanoglu I, Gul M, Kapan M, Arikanoglu Z, Taskesen F, Basol $\mathrm{O}$, et al. Risk factors effecting mortality in acute mesenteric ischemia and mortality rates: A single center experience. Int Surg 2013; 98:76-81.

17. Yildirim D, Hut A, Tatar C, Dönmez T, Akinci M, Toptas M. Prognostic factors in patients with acute mesenteric ischemia. Turk J Surg 2017; 33:104-9.

18. Angelone T, Filice E, Pasqua T, Amodio N, Galluccio M, Montesanti G, et al. Nesfatin-1 as a novel cardiac peptide: identification, functional characterization, and protection against ischemia/reperfusion injury. Cell Mol Life Sci 2013; 70:495-509

19. Jiang G, Wang M, Wang L, Chen H, Chen Z, Guo J, et al. The protective effect of nesfatin-1 against renal ischemiareperfusion injury in rats. Ren Fail 2015; 37:882-9.

20. Ayada C, Toru U, Genc O, Akcilar R, Sahin S. Balanced oxidative status by nesfatin-1 in intestinal ischemiareperfusion. Int J Clin Exp Med 2015; 15:3318-24.

21. Dai H, Li X, He T. Decreased plasma nesfatin-1 levels in patient with acute myokardial infarction. Peptides 2013; 46: 167-71.

22. Kolgazi M, Cantali-Ozturk C, Deniz R, Ozdemir-Kumral ZN, Yuksel $M$, Sirvanci $S$, et al. Nesfatin-1alleviates gastric damage via direct antioxidant mechanisms. J Surg Res 2015; 193:111-8.

23. Ozturk CC, Oktay S, Yuksel M, Akakin D, Yarat A, Kasimay Cakir O. Anti-inflammatory effects of nesfatin-1 in rats with acetic acid induced colitis and underlying mechanisms. J Phsysiol Pharmacol 2015; 66:741-50. 